\title{
Computer-assisted three-dimensional tracking of sensory innervation in the murine bladder mucosa with two-photon microscopy
}

Citation for published version (APA):

Schueth, A., Spronck, B., van Zandvoort, M. A. M. J., \& van Koeveringe, G. A. (2017). Computer-assisted three-dimensional tracking of sensory innervation in the murine bladder mucosa with two-photon microscopy. Journal of Chemical Neuroanatomy, 85, 43-49.

https://doi.org/10.1016/j.jchemneu.2017.06.006

Document status and date:

Published: 01/11/2017

DOI:

10.1016/j.jchemneu.2017.06.006

Document Version:

Publisher's PDF, also known as Version of record

Document license:

Taverne

Please check the document version of this publication:

- A submitted manuscript is the version of the article upon submission and before peer-review. There can be important differences between the submitted version and the official published version of record.

People interested in the research are advised to contact the author for the final version of the publication, or visit the DOI to the publisher's website.

- The final author version and the galley proof are versions of the publication after peer review.

- The final published version features the final layout of the paper including the volume, issue and page numbers.

Link to publication

\footnotetext{
General rights rights.

- You may freely distribute the URL identifying the publication in the public portal. please follow below link for the End User Agreement:

www.umlib.nl/taverne-license

Take down policy

If you believe that this document breaches copyright please contact us at:

repository@maastrichtuniversity.nl

providing details and we will investigate your claim.
}

Copyright and moral rights for the publications made accessible in the public portal are retained by the authors and/or other copyright owners and it is a condition of accessing publications that users recognise and abide by the legal requirements associated with these

- Users may download and print one copy of any publication from the public portal for the purpose of private study or research.

- You may not further distribute the material or use it for any profit-making activity or commercial gain

If the publication is distributed under the terms of Article $25 \mathrm{fa}$ of the Dutch Copyright Act, indicated by the "Taverne" license above, 


\title{
Computer-assisted three-dimensional tracking of sensory innervation in the murine bladder mucosa with two-photon microscopy
}

\author{
Anna Schueth ${ }^{\mathrm{a}, \mathrm{b}, *, 1}$, Bart Spronck ${ }^{\mathrm{c}, 1}$, Marc A.M.J. van Zandvoort ${ }^{\mathrm{d}, \mathrm{e}}$, \\ Gommert A. van Koeveringe ${ }^{a}$ \\ a Department of Urology, Maastricht University Medical Center, P.O. Box 5800, 6202 AZ, Maastricht, The Netherlands \\ bepartment of Cognitive Neuroscience, Maastricht Brain Imaging Center (MBIC), Maastricht University, Oxfordlaan 55, 6200 MD, Maastricht, The \\ Netherlands \\ ${ }^{\mathrm{c}}$ Department of Biomedical Engineering, CARIM School for Cardiovascular Diseases, Maastricht University, 6229 ER, Maastricht, The Netherlands \\ d Department of Genetics and Cell Biology - Molecular Cell Biology, CARIM School for Cardiovascular Diseases, Maastricht University, 6229 ER, Maastricht, \\ The Netherlands \\ e Institute for Molecular Cardiovascular Research (IMCAR), RWTH University of Aachen, Pauwelsstrasse 30, 52074 Aachen, Germany
}

\section{A R T I C L E I N F O}

\section{Article history:}

Received 20 April 2017

Received in revised form 23 June 2017

Accepted 26 June 2017

Available online 28 June 2017

\section{Keywords:}

Mice

Urinary bladder

3D nerve tracking

Lamina propria

3D microscopy

\section{A B S T R A C T}

A strong association between functional bladder disorders and bladder sensation is well-known, with a relationship between malfunctioning detrusor muscle and abnormal sensation arising from the suburothelium and the lamina propria (LP), has been suggested. However, the exact underlying pathophysiology of these bladder disorders is not completely understood. Therefore, it is important to gain knowledge on sensory innervation of the urinary bladder in order to understand the neural network function in healthy and diseased bladder. In the present study we aim at the development of a computer-assisted method for 3D-tracking of sensory innervation in the murine bladder mucosa using two-photon laser scanning microscopy (TPLSM). TPLSM was performed on 10 fixed, stained (CGRP) bladder samples in both the trigone and dome. Nerve tracking was performed in subvolumes $\left(6.3 \pm 2.910^{6} \mu^{3}\right.$; median $\left.\pm I Q R\right)$ of 22 stacks with determining total nerve length, nerve segment lengths, curviness, straightness, and locations of branching and ending points in the lamina propria (LP). The results show that the highest concentration of afferent fibres was found at the urothelium-LP interface. Nerve curviness, a presumed indicator of nerve activity, showed an equal value throughout the complete LP. We found a significantly higher median nerve segment length in the LP of the trigone and significantly more curved nerves in the dome of the bladder. This indicates an adaptation to, or an involvement in the detection of, bladder volume changes. Conclusively, we successfully developed a computer-assisted method for 3D tracking of sensory nerve fibres in the LP of the murine bladder wall. (C) 2017 Elsevier B.V. All rights reserved.

\section{Introduction}

Impairment of innervation of sensory pathways can be a cause of functional bladder disorders and results in storage or voiding dysfunction, such as the overactive bladder syndrome (OAB)

Abbreviations: CGRP, calcitonin gene-related peptide; $\mathrm{CO}$, connective tissue; KDE, kernel density estimate; LP, lamina propria; OAB, overactive bladder syndrome; ROI, region of interest; 3D, three-dimensional; TPLSM, two-photon laser scanning microscopy; $\mu \mathrm{m}$, micrometer.

* Corresponding author at: Department of Cognitive Neuroscience, Maastricht Brain Imaging Center (MBIC), Maastricht University, Oxfordlaan 55, 6200 MD, Maastricht, The Netherlands.

E-mail address: anna.schueth@maastrichtuniversity.nl (A. Schueth).

1 Contributed equally to this work.
(Natalin et al., 2013) or underactive bladder. A strong association between $O A B$ and bladder sensation is well-known, with a relationship between malfunctioning detrusor muscle and abnormal sensation arising from the sub-urothelium and the lamina propria (LP), having been suggested (Birder, 2013). However, the exact underlying pathophysiology of these bladder disorders is not completely understood. Therefore, it is important to gain knowledge on sensory innervation of the urinary bladder in order to understand the neural network function in healthy and diseased bladder.

It is known that afferent nerve fibres within the lower urinary tract (bladder and urethra) originate from pelvic, pudendal, and hypogastric nerves. They carry sensory information on the filling status of the bladder to the spinal cord, where they subsequently initiate the activation of brain areas involved in regulation of 
micturition (Kanai and Andersson, 2010). It is generally accepted that there are two different populations of afferent nerve fibres in the urinary tract: myelinated $A \delta$ and unmyelinated C-fibres (de Groat et al., 2015). The sensitive A $\delta$ fibres are located mainly in the muscle layer of the bladder wall and communicate during bladder filling via mechano-transduction to the central nervous system (Kanai and Andersson, 2010; Andersson, 2011). Under healthy conditions, the unmyelinated, "silent" C-fibres are present but minimally active in the muscle layer, the LP, and sub-urothelium of the bladder wall (Kanai and Andersson, 2010). They can become active in several pathological situations where they respond mostly to noxious stimuli, such as harmful chemicals or inflammation (Cheng et al., 1993; Habler et al., 1990). Xu and Gebhart described four different subtypes of C-fibres in the murine bladder, based on their responses to mechanical stimuli (in vitro study): serosal, muscular, muscular/urothelial, and urothelial afferents (Xu and Gebhart, 2008). Muscular, muscular/urothelial, and serosal afferents were found as lumbar splanchnic nerves, whereas all four types were found as sacral pelvic nerves (Xu and Gebhart, 2008).

To date, studies on sensory innervation of the bladder wall, including quantitative analyses of nerve distribution, are very limited or have not been updated for many years. In 1998, Gabella and Davis examined the afferent nerve distribution in rat bladder samples using classical immunohistochemistry and confocal microscopy (Gabella and Davis, 1998), providing only twodimensional information. Additional and new insights in nerve pathways by means of three-dimensional (3D) reconstructions could enable the study of e.g. distribution and shape of nerves within the entire organ. As one example, the application of twophoton laser scanning microscopy (TPLSM) to image the transgenic mouse heart revealed the 3D microarchitecture of the sympathetic innervation therein (Freeman et al., 2014). Similarly, the use of TPLSM could potentially yield information on the three-dimensional distribution and orientation of afferent nerve fibres within the bladder wall.

In the present study, we will use TPLSM as a new approach to investigate the 3D structure of afferent nerves within the murine urinary bladder. In a previous TPLSM study, we semi-quantitatively studied the morphological changes in the bladder wall architecture occurring with age, such as the number of nerve fibres and the ratio between collagen and smooth muscle volumes (Schueth et al., 2016). We will continue in line with that research by developing a computer-assisted method for 3D tracking of sensory innervation in the murine bladder wall. Moreover, we aim to quantitatively and objectively analyse distribution, density, length, and shape of afferent nerve fibres as identified by immunohistochemical staining for calcitonin gene-related peptide (CGRP) in the LP of the murine bladder wall. CGRP positive nerves have been shown to form a major part of the afferent nervous circuitry in the bladder of several animal models and even humans. CGRP nerves are thought to play a modulatory role as they are often found in close proximity to motor ganglia and nerves. Therefore these afferent nerves are likely to play a major role in the motor-sensory control of the urinary bladder. To the best of our knowledge, this is the first report of automated TPLSM-3D tracking of sensory nerves in the murine bladder wall.

\section{Materials and methods}

\subsection{Animals}

Experimental protocols were approved by the animal experiments committee of Maastricht University, and were carried out according to institutional guidelines and reported in accordance with the ARRIVE guidelines. Ten C57BL/6J wild-type mice (age range between 10 and 25 weeks) were housed in individuallyventilated cages within a temperature-controlled environment with 12-h light/dark cycle, standard chow, and water available ad libitum.

\subsection{Tissue preparation and immunohistochemical staining}

Nerves were stained with CGRP (Anti-Calcitonin Gene Related Peptide), Rabbit pAB (Merck) with Alexa 594 secondary antibody (Life Technologies $^{\mathrm{TM}}$ ). Fixed bladder tissue was washed 3 times with Tris-Buffered Saline (TBS) (Merck), each for $15 \mathrm{~min}$. Primary antibody (CGRP) was diluted 1:1000 in TBS-Triton (0,3\%), applied to the tissue, and incubated overnight at $4{ }^{\circ} \mathrm{C}$. The primary antibody was removed and the sample was washed 3 times, each for $30 \mathrm{~min}$ with TBS-Triton (0,3\%), TBS and TBS-Triton (0,3\%), respectively. Secondary antibody (Alexa 594) was diluted 1:100 in TBS-Triton $(0,3 \%)$, applied to the sample and incubated in the dark, overnight at $4{ }^{\circ} \mathrm{C}$. The secondary antibody was removed and the sample was washed 3 times, each for $15 \mathrm{~min}$, with TBS-Triton (0,3\%), TBS and TBS, respectively

During TPLSM imaging the prepared bladders were placed in a petri dish and submerged under saline solution (Schueth et al., 2014). Images were taken from the urothelial side. In each bladder, both dome and trigone regions were imaged.

\subsection{Imaging}

For imaging experiments, a two-photon laser scanning microscope (Leica TCS SP5 MP, Leica Mikrosysteme Vertrieb GmbH, Wetzlar, Germany), equipped with a HCX APO L $20 \times / 1.00 \mathrm{~W}$ water immersion objective was used. Working distance of the objective was $2 \mathrm{~mm}$ and the excitation source was a $140 \mathrm{fs}$-pulsed Ti: sapphire laser (Chameleon Ultra II, Coherent Inc., Santa Clara, CA, USA), mode-locked at $800 \mathrm{~nm}$. To avoid photobleaching and tissue damage, laser power was kept as low as possible (max. 100$125 \mathrm{~mW}$, at the sample surface). Fluorescence emission was detected using photomultiplier tubes (Hamamatsu, R9624, Japan) in three wavelength ranges:

1. $385-489 \mathrm{~nm}$ (blue), typically showing collagen fibres as a result of second harmonic generation (Megens et al., 2007),

2. $489-563 \mathrm{~nm}$ (green), acquiring the autofluorescence signal of e.g. umbrella cells, and elastic fibres

3. $568-700 \mathrm{~nm}$ (red), acquiring the CGRP-Alexa 594 signal of stained afferent fibres.

Images and image stacks were acquired using 12-bit precision with Leica Application Suite Advanced Fluorescence (Leica Microsystems). Image acquisition settings for the experiments are

Table 1

TPLSM image acquisition settings for fixed bladder samples.

\begin{tabular}{|c|c|c|c|c|c|c|}
\hline mode & scan field resolution & scan field size $[\mu \mathrm{m} \times \mu \mathrm{m}]$ & pixel size $[\mu \mathrm{m}]$ & line rate $[\mathrm{Hz}]$ & line averaging & frame rate $[\mathrm{Hz}]$ \\
\hline examination & $512 \times 512$ & $738 \times 738$ & 1.4 & 400 & 1 & 0.8 \\
\hline single slice and stack recording & $1024 \times 1024$ & $738 \times 738$ & 1.4 & 100 or 200 & 2 & $0-0.2$ \\
\hline
\end{tabular}

Stacks were recorded with a slice spacing ("z-step") of $1 \mu \mathrm{m}$. 
elaborated in Table 1. The use of these developed image settings, which are specific for fixed and stained bladder samples, control the quality of the images for later nerve tracking, and decrease photobleaching and any damage to the tissue.

\subsection{Nerve tracking of stained nerve fibres using IMARIS filament tracer}

Raw, unprocessed image stacks were loaded into Imaris (Bitplane AG, Zürich, Switzerland). Nerve tracking was performed using the FilamentTracer package in subvolumes $\left(6.3 \pm 2.910^{6} \mu \mathrm{m}^{3} ;\right.$ median $\left.\pm \mathrm{IQR}\right)$ of the original image stacks. After setting a region of interest (ROI) and displaying in display adjustment only the red channel dendrites, beginning points, branch points, and terminal points were chosen for measurements. The selected base colour for dendrites is indicated in yellow. Subsequently, thresholds for start and seeding points are set to be determined automatically ("automated"), and the threshold detection type for the dendrites is set to "local contrast".

\subsection{Morphological quantification of tracked nerves}

After nerve tracking, coordinates of the traced nerve segments were directly imported from Imaris into MATLAB $^{\circledR}$ R2015b (MathWorks ${ }^{\circledR}$, Natick, MA). A segment is defined as non-branching part of a nerve tracing. Therefore, segments start and end in either a branching point or an end point.

A segment end point typically indicates the location of a nerve ending, provided the end point is not at the border of the nerve tracing subvolume. Therefore, nerve endings at the tracing subvolume borders were excluded from our analyses.

In order to determine nerve segment length and curvature, a periodic interpolating cubic spline curve $\vec{r}(t)$ was fitted to each segment using the MATLAB Curve Fitting Toolbox function cscvn, with

$$
\vec{r}(t)=\langle\mathrm{f}(t), \mathrm{g}(t), \mathrm{h}(t)\rangle
$$

in which $f(t), g(t)$, and $h(t)$ are the $\mathrm{x}, \mathrm{y}$, and $\mathrm{z}$ coordinates of points along a segment.
Segment length $(L)$ can now be determined by numerical integration of $\left\|r^{\prime}(t)\right\|$ :

$$
L=\int_{a}^{b}\left\|r^{\prime}(t)\right\| \mathrm{d} t,
$$

with $a$ and $b$ the start and end $t^{\prime}$ s of the segment, and $\vec{r}^{\prime}(t)$ denoting $\partial \vec{r} / \partial t$.

Curvature $\kappa\left[\mu \mathrm{m}^{-1}\right]$ is subsequently calculated as

$$
\kappa=\| \frac{r^{\prime}(t) \cdot \vec{r}^{\prime \prime}(t) \|}{\left\|r^{\prime}(t)\right\|^{3},}
$$

with $\vec{r}^{\prime \prime}(t)$ denoting $\partial^{2} \vec{r} / \partial t^{2}$.

Curvature is related to the local radius of curvature $(R(t),[\mu \mathrm{m}])$ via

$\kappa=\frac{1}{R(t)}$.

In this paper, we have chosen to report curvature, and not radius of curvature, since for relatively straight nerves, $R(t)$ goes to infinity, causing problems in calculating proper summary statistical measures. Curvature is a measure for curvature.

In addition to curvature, we also computed straightness as a measure of curvature of the nerves. Straightness $(W)$ is defined as $W=\frac{L_{\mathrm{direct}}}{L}$,

where $L_{\text {direct }}$ is the straight-line distance between a nerve segment's start and end points. Therefore, straightness is a measure of straightness: the higher its value, the straighter the nerve segment.

Although both straightness and curvature provide a means to quantify nerve curvature, they are conceptually different. Whereas curvature quantifies the local curvature of a nerve, straightness quantifies the curvature of an entire nerve segment.

\subsection{Statistics}

All values are presented as median \pm interquartile range (IQR). Group comparisons were made using non-paramatric Wilcoxon
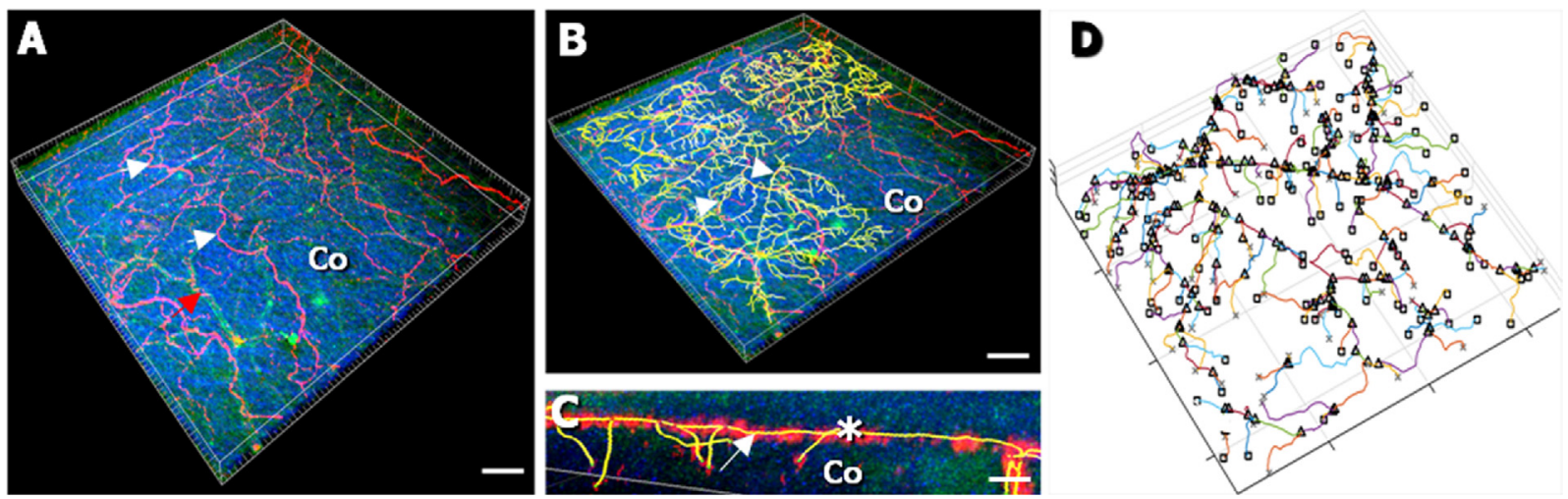

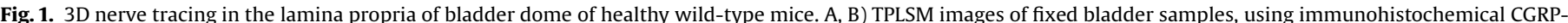

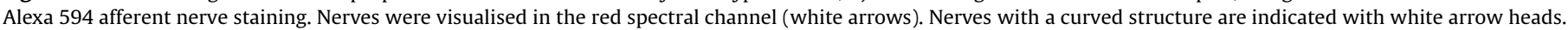

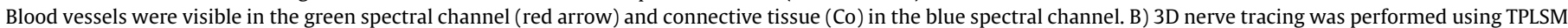

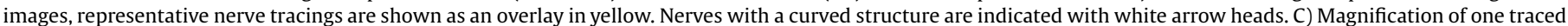

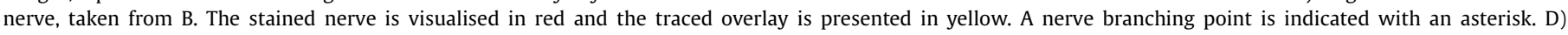

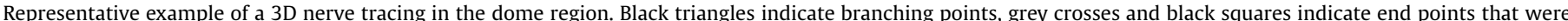

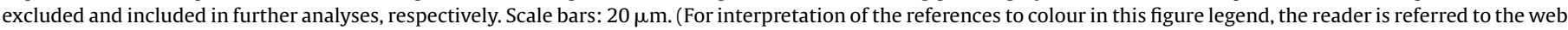
version of this article.) 
rank-sum tests. P-values $<0.05$ were considered statistically significant.

\subsection{Kernel density estimation}

We described the use of kernel density estimation in an equivalent setting previously (Spronck et al., 2014). Briefly, for each stack, imaging depth was normalized from 0 to 1 , after which a two-dimensional kernel density estimate (KDE) was calculated. Kernel density estimation allows for estimation and visualization of the probability density from a set of data points. In our case, the KDE shows how often a certain curvature or straightness is encountered at a certain imaging depth. For each depth, four quantiles (quartiles) were calculated and displayed as lines on the KDE. In addition to the KDEs per stack (as exemplified in Fig. 2EF), an overall KDE was calculated of all 22 acquired stacks.

\section{Results}

3.1. Tracking of afferent nerve fibres in the lamina propria of the murine bladder wall

Nerve tracing was performed in subvolumes $\left(6.3 \pm 2.910^{6} \mu \mathrm{m}^{3}\right.$; median $\pm \mathrm{IQR}$ ) of 22 image stacks, which were obtained from 10 mice. Four of the 22 processed image stacks were acquired in the trigone region of the bladder, whereas the other 18 stacks were acquired in the dome region.

In each stack we determined total nerve length, nerve segment lengths, curvature, straightness, and locations of branching and ending points. A representative example of such a nerve tracing is shown in Fig. 1B,C. Features detected in this stack are summarised in Fig. 2A-F. Summary statistics for the 22 image stacks are presented in Table 2.
Table 2

Summary of nerve tracing results from 22 image stacks.

\begin{tabular}{lllll}
\hline parameter & unit & median & & IQR \\
\hline total nerve length & $\mu \mathrm{m} /\left(10^{6} \mu \mathrm{m}^{3}\right)$ & 1297 & \pm & 664 \\
segment length & $\mu \mathrm{m}$ & 20 & \pm & 5 \\
number of branching points & $\# /\left(10^{6} \mu \mathrm{m}^{3}\right)$ & 23 & \pm & 20 \\
number of ending points & $\# /\left(10^{6} \mu \mathrm{m}^{3}\right)$ & 18 & \pm & 16 \\
curviness & $\mu \mathrm{m}^{-1}$ & 0.131 & \pm & 0.014 \\
straightness & - & 0.881 & \pm & 0.085 \\
\hline
\end{tabular}

$\mathrm{IQR}$, interquartile range.

\subsection{Quantification and analysis of nerve length, shape, branching and} end points

Median nerve segment length was $23 \pm 11$ (Fig. 3D). In all image stacks, we found a relatively high nerve concentration at the interface between the LP and the urothelium (Fig. 3C; peak at low imaging depth). While analysing the branching points and end points of the tracked nerves, we found them to be spread out in depth in the LP, with branching points clearly showing a peak at the LP-urothelium transition (Fig. $3 \mathrm{~A}, \mathrm{~B}$ ), and end points showing a maximum approximately halfway the LP. Curvature, a local measure of nerve curvature, was evenly distributed across all imaging depths (Fig. 3B). Straightness, a more global curvature measure that quantifies the straightness of a nerve segments in one number, also showed no consistent fluctuation with depth (Fig. 3F).

\subsection{Comparison of sensory innervation in the bladder dome vs. trigone}

We compared summary statistical measures from the dome and trigone regions. Results are presented in Table 3. Median nerve segment length in the trigone was slightly, yet significantly, larger
A

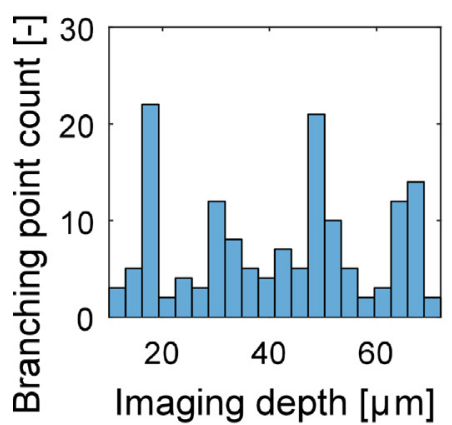

D

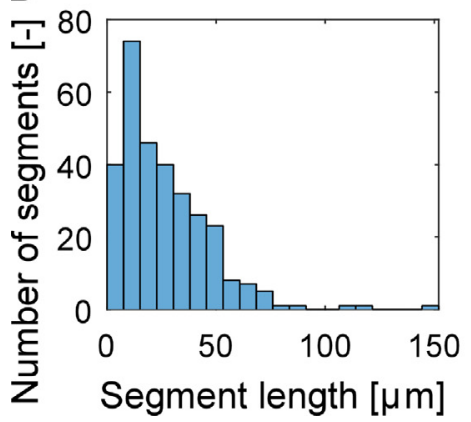

B

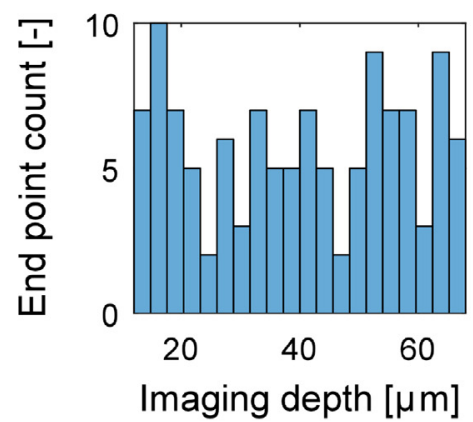

E

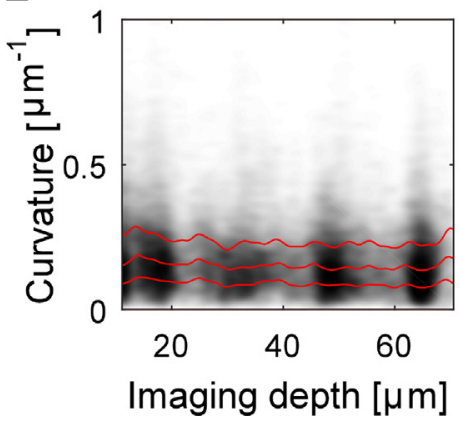

C

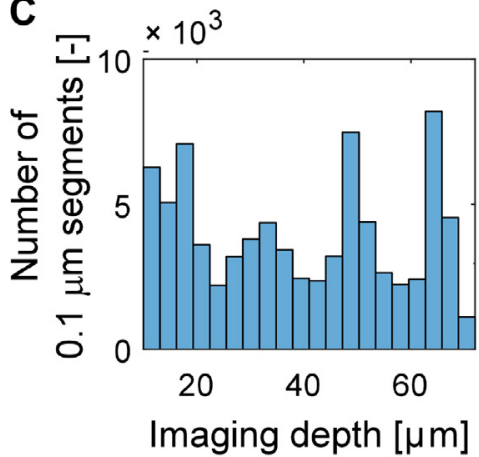

$\mathbf{F}$

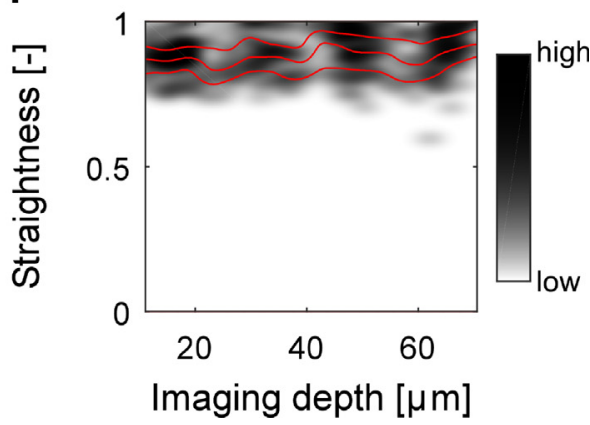

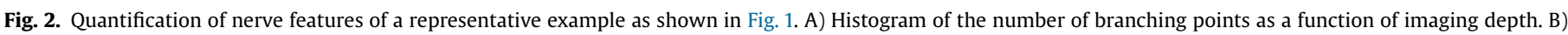

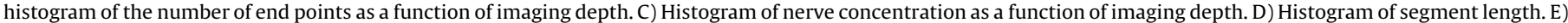

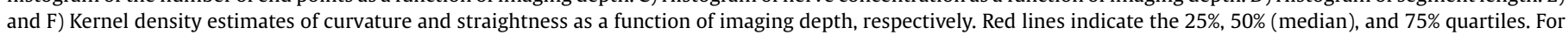
processing details, see text. (For interpretation of the references to colour in this figure legend, the reader is referred to the web version of this article.) 
A
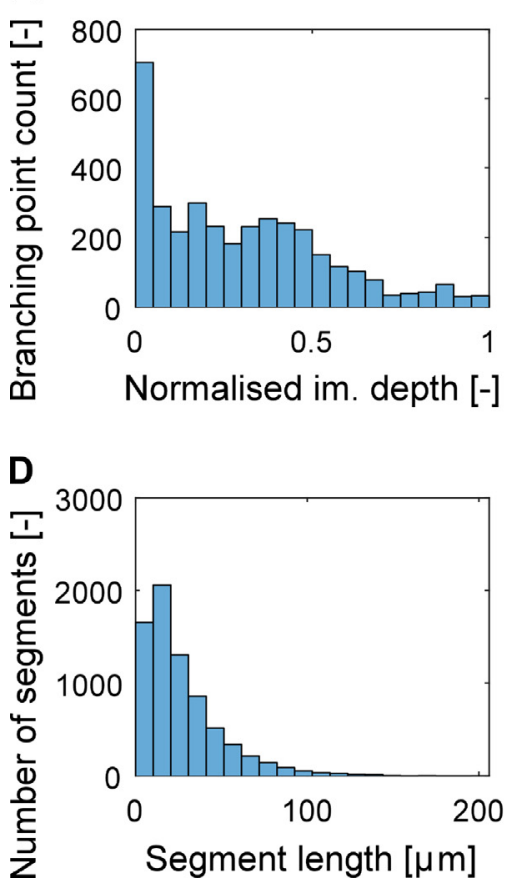

B

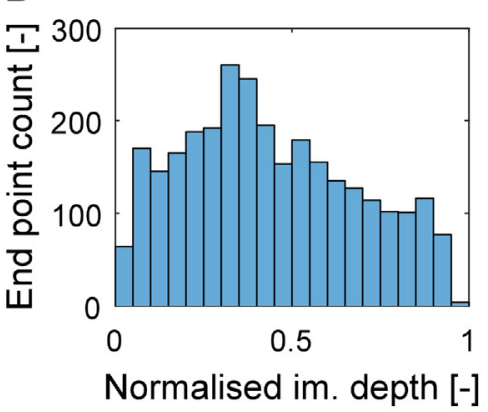

E

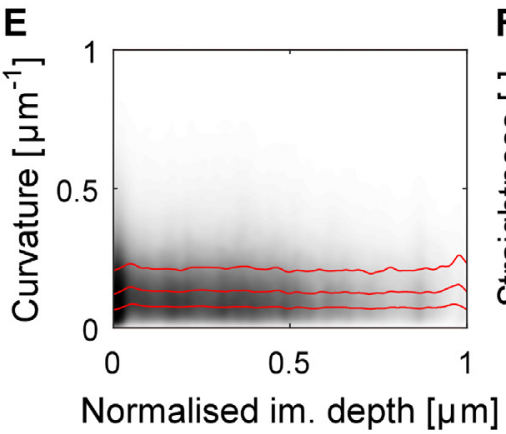

C
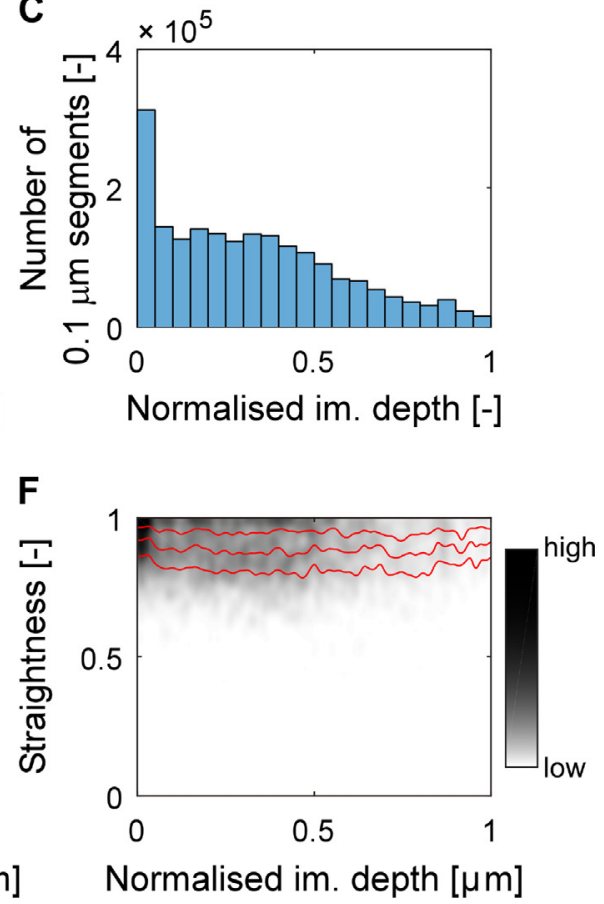

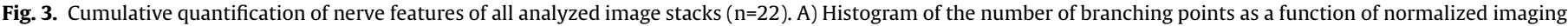

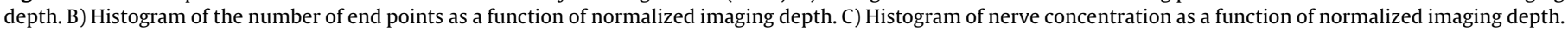

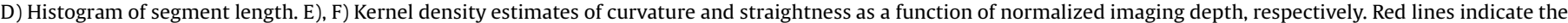

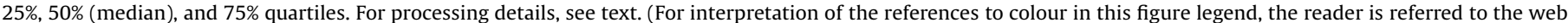
version of this article.)

Table 3

Nerve tracing results stratified to trigone and dome regions.

\begin{tabular}{|c|c|c|c|c|c|c|c|c|}
\hline \multirow{8}{*}{$\begin{array}{l}\text { parameter } \\
\text { total nerve length } \\
\text { segment length } \\
\text { number of branching points } \\
\text { number of ending points } \\
\text { curviness } \\
\text { straightness }\end{array}$} & \multirow{3}{*}{$\begin{array}{l}\text { unit } \\
\mu \mathrm{m} /\left(10^{6} \mu \mathrm{m}^{3}\right)\end{array}$} & \multicolumn{3}{|c|}{ trigone $(n=4)$} & \multicolumn{4}{|c|}{ dome $(n=18)$} \\
\hline & & median & & IQR & median & & IQR & $p$ \\
\hline & & 983 & \pm & 183 & 1407 & \pm & 832 & 0.327 \\
\hline & $\mu \mathrm{m}$ & 24 & \pm & 1 & 20 & \pm & 4 & 0.045 \\
\hline & $\# /\left(10^{6} \mu \mathrm{m}^{3}\right)$ & 15 & \pm & 3 & 27 & \pm & 22 & 0.115 \\
\hline & $\# /\left(10^{6} \mu \mathrm{m}^{3}\right)$ & 13 & \pm & 3 & 22 & \pm & 14 & 0.081 \\
\hline & $\mu \mathrm{m}^{-1}$ & 0.121 & \pm & 0.002 & 0.132 & \pm & 0.012 & 0.006 \\
\hline & - & 0.879 & \pm & 0.114 & 0.881 & \pm & 0.084 & 0.831 \\
\hline
\end{tabular}

$\mathrm{IQR}$, interquartile range; $p, p$-value of Wilcoxon rank-sum test comparing trigone to dome.

than in the dome $(24 \pm 1$ vs. $20 \pm 4 \mu \mathrm{m}, p=0.045)$. Correspondingly, albeit not statistically significantly, there were on average more branching points in the dome than in the trigone ( $27 \pm 22 \mathrm{vs} .15 \pm 3$ per $\left.10^{6} \mu \mathrm{m}, p=0.115\right)$. Curvature in the trigone was slightly, but significantly, smaller than in the dome $\left(0.121 \pm 0.002 \mu \mathrm{m}^{-1} \mathrm{vs}\right.$. $0.132 \pm 0.012 \mu \mathrm{m}^{-1}, p=0.006$ ), indicating that in the dome region nerves were locally more curved than in the trigone region. Straightness did not differ between trigone and dome $(0.879 \pm 0.114$ vs. $0.881 \pm 0.084, p=0.831)$.

\section{Discussion}

4.1. Tracking of afferent nerve fibres in the lamina propria of the murine bladder wall

For storage and expulsion of urine, afferent nerves communicate and monitor the bladder volume, and their function is necessary to subsequently initiate and probably maintain the micturition reflex (Kanai and Andersson, 2010). Although it has been described that afferent nerve fibres are distributed in both the LP (Gabella and Davis, 1998) and in the detrusor muscle of the bladder wall (Andersson, 2002), these studies lack information on bladder innervation in three dimensions. With our development of a computer-assisted method for 3D nerve tracking in the LP of the murine bladder wall, we significantly improve upon the already existing studies on afferent nerve distribution.

\subsection{Quantification and analysis of nerve length, shape, branching and end points}

The general distribution of afferent fibres in the rat bladder was studied in 1998 by Gabella and Davis using CGRP-immunohistochemical staining together with classic fluorescence microscopy (Gabella and Davis, 1998). Afferents were found in the LP and the urothelium, and both along muscle bundles and blood vessels (Gabella and Davis, 1998). The serosal layer of the rat bladder was lacking in afferent fibres (Gabella and Davis, 1998).

In our study, we showed that the afferent nerve fibres (stained with CGRP immuno-histochemistry) were distributed throughout the entire LP, with the highest concentration of nerves, nerve branching, and end points at the interface between LP and the urothelium. This phenomenon goes in line with the finding of a 
sub-urothelial plexus of afferent nerve fibres (Andersson, 2002). It has been suggested that this "sensory web" acts a signal transducer, which transmits information on bladder filling to the underlying detrusor (Andersson, 2002). It is known that afferent nerves located in the LP detect information on the bladder filling status as well as pain signals and convey this information via the spinal cord to the responsible areas in the brain (Andersson and McCloskey, 2014; Fowler et al., 2008). The urothelium, above the LP, detects changes in its extracellular environment, and responds to e.g. mechanical stimuli with neurotransmitter release (Birder and Andersson, 2013). Thus, the LP and the sensory innervation therein play a crucial role in the micturition process and are therefore of high importance for proper bladder function (Andersson and McCloskey, 2014). However, the role of the LP is still under-researched and not fully established yet (Andersson and McCloskey, 2014).

In our analyses, nerve curvature and straightness did not vary much with imaging depth. It is generally accepted that (efferent) nerves modify their straight shape into a curved shape when they release neurotransmitter and get activated (Andersson, 2015; Burnstock, 2014). In the present study, we can only speculate whether this activation was also applicable to the curved afferent nerves we saw. It may very well be that the transition from curved to stretched involves a detection mechanism for bladder filling. Our finding could be another indicator for the role of the LP as signal transducer and communication centre, as it has been suggested earlier (Andersson and McCloskey, 2014). Moreover, this could suggest a role of the murine LP in volume sensation. Although with our experimental set-up it was not possible to assess dynamic processes of nerves, future in vivo studies might give the answer to this hypothesis.

Furthermore, we detected afferent fibres, but we can only speculate whether these are A $\delta$ or C-fibres. Four sub-classes of Cfibres in the murine bladder have been described by $\mathrm{Xu}$ and Gebhart, based on their responses to mechanical stimuli: serosal, muscular, muscular/urothelial, and urothelial afferents (Xu and Gebhart, 2008). In addition, mainly "silent" C-fibres are present in the LP, which respond to an injury (Iijima et al., 2009; de Groat and Yoshimura, 2010) or inflammation (Yoshimura and de Groat, 1999). Some evidence has been found that also high-threshold C-fibres (like the myelinated A $\delta$ fibres) are involved in the normal sensing process bladder filling (Yoshimura and de Groat, 1999). In addition, Fowler described that after spinal disruption an alternative afferent signalling pathway with C-fibres evokes (spinal segmental reflex pathway), whereas normally A $\delta$ fibres play the cardinal role in transmitting the bladder filling state to the brain (Fowler, 2002). In humans, A $\mathrm{\delta}$ fibres can be activated by low bladder pressure of approximately $5-15 \mathrm{~mm} \mathrm{Hg}$, which is in many cases the first sensation of bladder filling (Andersson, 2011; Rong et al., 2002). Recently, Danziger and Grill described that afferent fibres also detect bladder pressure or detrusor stretch, rather than bladder filling (Danziger and Grill, 2016).

\subsection{Comparison of sensory innervation in the bladder dome vs. trigone}

The trigone of the urinary bladder wall is the triangular region located between the two ureteral orifices (left and right) and the bladder outlet (urethra) (Bortolini et al., 2014). During bladder filling, the trigone contracts, the ureteral orifices open up and the bladder neck is closed. On the other hand, during micturition the trigone relaxes and allows the expulsion of urine through the urethra (Bortolini et al., 2014).

In our study, we detected that in the trigone of the murine bladder wall, the median nerve segment length was slightly larger than in the dome. This finding goes in line with the fact that the afferent fibres are notably dense in the bladder neck and the trigone, especially in the sub-urothelial plexus (Andersson, 2002).

Furthermore, Gabella and Davis described that the afferent nerve density was the highest at the bladder neck region in the rat, whereas the dome was only sporadically innervated (Gabella and Davis, 1998).

The dome is the biggest part of the bladder and has a strong contracting ability due to its thick smooth muscle layer (Sadananda et al., 2008), which is crucial for the micturition process. Although not statistically significant, we found more branching points in the dome than in the trigone. One explanation for this could be that afferent signals have to be detected over a bigger surface than in the trigone. In addition, we showed that the afferent nerves in the bladder dome were slightly, but significantly more curved as compared to the nerves in the trigone. This might be an indication for the dome being more involved in large surface area changes compared to the trigone. Another possible explanation could be, that the trigone has a relatively stable shape during the bladder filling process as opposed to the dome of the bladder, which is the most distensible part. Furthermore, Danziger and Grill, suggested that the bladder dome detects large distension, in contrast to moderate distension of the bladder neck, and that results in a higher presence of afferents in the dome (Danziger and Grill, 2016).

We showed that the method we developed is suitable for nerve quantification in the bladder wall of healthy wild-type mice. However, in the present study we have examined the LP only. In a follow-up study, inclusion of the smooth muscle layer of the bladder wall could potentially allow us to compare A $\delta$ fibres, which are present in the detrusor and have an important mechanotransduction function, with C-fibres. For this we would perform additional stainings that differentiate between unmyelinated and myelinated afferent fibres. Moreover, functional experiments, such as mechanical stimulation, (e.g. von Frey probing or stretching), may allow to find a distinction between sub-classes of C-fibres. This could improve our understanding of the sensory innervation of the bladder wall and eventually help to improve the insight into bladder dysfunctions (incontinence and urgency) with impaired afferent signalling.

\section{Conclusions}

We successfully developed a computer-assisted method for 3D tracking of afferent nerve fibres in the LP of the bladder wall in healthy wild-type mice. We detected afferent fibres throughout the entire LP, with the highest concentration of nerves, nerve branching, and end points at the interface between LP and the urothelium. Moreover, nerve curvature, a presumed indicator of nerve activity, showed an equal value throughout the complete LP. Comparing LP innervation in the bladder dome and the trigone, we found a significantly higher median nerve segment length in the trigone. Also, we found significantly more curved nerves in the dome of the bladder, which indicates an adaptation to, or an involvement in the detection of bladder volume changes.

\section{Acknowledgements}

The EU - TRUST FP7-Marie Curie program and the WAMU foundation Maastricht has funded the research. We thank Jeroen Hameleers for his excellent technical support.

\section{References}

Andersson, K.E., McCloskey, K.D., 2014. Lamina propria: the functional center of the bladder? Neurourol. Urodyn. 33, 9.

Andersson, K.E., 2002. Bladder activation: afferent mechanisms. Urology 59, 43. Andersson, K.E.M., 2011. M.C.: Urinary Tract. Springer, Heidelberg. 
Andersson, K.E., 2015. Purinergic signalling in the urinary bladder. Auton. Neurosci. $191,78$.

Birder, L., Andersson, K.E., 2013. Urothelial signaling. Physiol. Rev. 93, 653.

Birder, L.A., 2013. Nervous network for lower urinary tract function. Int. J. Urol. 20 (4) .

Bortolini, M.A., Bilhar, A.P., Castro, R.A., 2014. Neural control of lower urinary tract and targets for pharmacological therapy. Int. Urogynecol. J. 25, 1453.

Burnstock, G., 2014. Purinergic signalling in the urinary tract in health and disease. Purinergic Signal. 10, 103.

Cheng, C.L., Ma, C.P., de Groat, W.C., 1993. Effects of capsaicin on micturition and associated reflexes in rats. Am. J. Physiol. 265, R132.

Danziger, Z.C., Grill, W.M., 2016. Sensory and circuit mechanisms mediating lower urinary tract reflexes. Auton. Neurosci. 200 (October), 21-28. doi:http://dx.doi. org/10.1016/j.autneu.2015.06.004 Epub 2015 Jun 6.

Fowler, C.J., Griffiths, D., de Groat, W.C., 2008. The neural control of micturition. Nat. Rev. Neurosci. 9, 453.

Fowler, C.J., 2002. Bladder afferents and their role in the overactive bladder. Urology $59,37$.

Freeman, K., Tao, W., Sun, H., et al., 2014. In situ three-dimensional reconstruction of mouse heart sympathetic innervation by two-photon excitation fluorescence imaging. J. Neurosci. Methods 221, 48.

Gabella, G., Davis, C., 1998. Distribution of afferent axons in the bladder of rats. J. Neurocytol. 27, 141

Habler, H.J., Janig, W., Koltzenburg, M., 1990. Activation of unmyelinated afferent fibres by mechanical stimuli and inflammation of the urinary bladder in the cat. J. Physiol. 425, 545.

Iijima, K., Igawa, Y., Wyndaele, J.J., et al., 2009. Mechanosensitive primary bladder afferent activity in rats with and without spinal cord transection. J. Urol. 182, 2504.
Kanai, A., Andersson, K.E., 2010. Bladder afferent signaling: recent findings. J. Urol. $183,1288$.

Megens, R.T., Reitsma, S., Schiffers, P.H., et al., 2007. Two-photon microscopy of vital murine elastic and muscular arteries. Combined structural and functional imaging with subcellular resolution. J. Vasc. Res. 44, 87.

Natalin, R., Lorenzetti, F., Dambros, M., 2013. Management of OAB in those over age 65. Curr. Urol. Rep. 14, 379.

Rong, W., Spyer, K.M., Burnstock, G., 2002. Activation and sensitisation of low and high threshold afferent fibres mediated by P2X receptors in the mouse urinary bladder. J. Physiol. 541, 591.

Sadananda, P., Chess-Williams, R., Burcher, E., 2008. Contractile properties of the pig bladder mucosa in response to neurokinin A: a role for myofibroblasts? Br. J. Pharmacol. 153, 1465.

Schueth, A., van Zandvoort, M.A., Buurman, W.A., et al., 2014. Murine bladder imaging by 2-photon microscopy: an experimental study of morphology. J. Urol. 192, 973.

Schueth, A., Spronck, B., van Zandvoort, M.A., et al., 2016. Age-related changes in murine bladder structure and sensory innervation: a multiphoton microscopy quantitative analysis. Age (Dordr) 38, 17.

Spronck, B., Merken, J.J., Reesink, K.D., et al., 2014. Ureter smooth muscle cell orientation in rat is predominantly longitudinal. PLoS One 9, e86207.

Xu, L., Gebhart, G.F., 2008. Characterization of mouse lumbar splanchnic and pelvic nerve urinary bladder mechanosensory afferents. J. Neurophysiol. 99, 244.

Yoshimura, N., de Groat, W.C., 1999. Increased excitability of afferent neurons innervating rat urinary bladder after chronic bladder inflammation. J. Neurosci. $19,4644$.

de Groat, W.C., Yoshimura, N., 2010. Changes in afferent activity after spinal cord injury. Neurourol. Urodyn. 29, 63.

de Groat, W.C., Griffiths, D., Yoshimura, N., 2015. Neural control of the lower urinary tract. Compr. Physiol. 5, 327. 